\title{
Abordaje hemodinámico y ventilatorio en pacientes con COVID-19
} Hemodynamic and mechanical ventilation approach in patients with COVID-19

\begin{abstract}
Raúl Carrillo-Esper', Leslian Mejía-Gómez², Enrique Monares-Zepeda³, Uriel Chavarría-Martínez, Alejandro Díaz-Carrillo ${ }^{5}$, Miguel Ayala-León ${ }^{6}$, Sergio Zamora-Gómez ${ }^{7}$ Jesús S. Sánchez-Díaz ${ }^{8}$, Manuel Lomelí-Terán ${ }^{9}$, Jesús C. Briones-Garduño ${ }^{1}$ y Ángel A. Pérez-Calatayud ${ }^{10 *}$

${ }^{1}$ Academia Mexicana de Cirugía, Ciudad de México; ${ }^{2}$ Servicio de Anestesiología, Unidad Médica de Alta Especialidad, Hospital de Traumatología y Ortopedia, Lomas Verdes, Estado de México; ${ }^{3}$ Terapia Intensiva, Centro Médico ABC, Ciudad de México; ${ }^{4}$ Departamento de Terapia Intensiva, Hospitales Cristus Muguerza, Monterrey, Nuevo León; ${ }^{5}$ Departamento de terapia Intensiva, Hospital de Especialidades del Niño y la Mujer Dr. Felipe Núñez Lara, Santiago de Querétaro, Querétaro; ${ }^{6}$ Servicio de Terapia Intensiva Cardiovascular, Hospital Beneficencia Española, Puebla, Puebla; 'Departamento de Medicina Crítica, Hospital Beneficencia Española, Tampico, Tamaulipas; ${ }^{8}$ Unidad de Cuidados Intensivos Adultos, Hospital de Especialidades núm. 14, Centro Médico Nacional Adolfo Ruiz Cortines, Instituto Mexicano del Seguro Social, Veracruz, Veracruz; ${ }^{9}$ Áreas Críticas Hospital H+ Querétaro, Santiago de Querétaro, Querétaro; ${ }^{10}$ División de Áreas Críticas, Hospital General de México Dr. Eduardo Liceaga, Ciudad de México. México
\end{abstract}

\section{Resumen}

El coronavirus del síndrome respiratorio agudo grave 2 (SARS-CoV-2, conocido previamente como nCoV-2019) es el agente causal de una nueva enfermedad denominada COVID-19 (COronaVIrus Disease 2019) por la Organización Mundial de la Salud. Su presentación es la insuficiencia respiratoria aguda caracterizada por una hiperinflación del pulmón que conduce a un incremento de los capilares y permeabilidad epitelial, con pérdida de la aireación de tejido pulmonar e incremento de la rigidez pulmonar. Estas alteraciones conducen a desequilibrios entre la ventilación y la relación de perfusión, que finalmente resultan en hipoxemia y deterioro de la depuración de dióxido de carbono. Para la presente revisión se realizó una búsqueda en PubMed y Trip Database. Debido a la escasez de publicaciones no se utilizó un algoritmo de búsqueda específico. El objetivo es dar a conocer, de acuerdo con la evidencia y las recomendaciones de expertos nacionales e internacionales, el manejo hemodinámico y ventilatorio de estos pacientes.

Palabras clave: COVID-19. Hemodinamia. SARS-CoV-2. Ventilación mecánica.

\begin{abstract}
The severe acute respiratory syndrome (SARS-Cov-2) is a clinical entity generated by this new virus a Coronavirus (COVID-19). Disease called COVID-19 (CoronaVIrus Disease 2019) by the World Health Organization. Its presentation is acute respiratory failure characterized by hyperinflation of the lung that leads to an increase in capillaries and epithelial permeability, with loss of ventilation of lung tissue and increases lung stiffness. These disturbances lead to imbalances between ventilation and perfusion ratio, which ultimately result in hypoxemia and impaired carbon dioxide clearance. For this review, a search of PubMed and Trip Database was performed. Due to the scarcity of publications, a specific search algorithm was not used. The objective is to review, the evidence and the recommendations of national and international experts, of the hemodynamic and ventilatory management of these patients.
\end{abstract}

Key words: COVID-19. Hemodynamics. SARS- CoV-2. Mechanical ventilation.

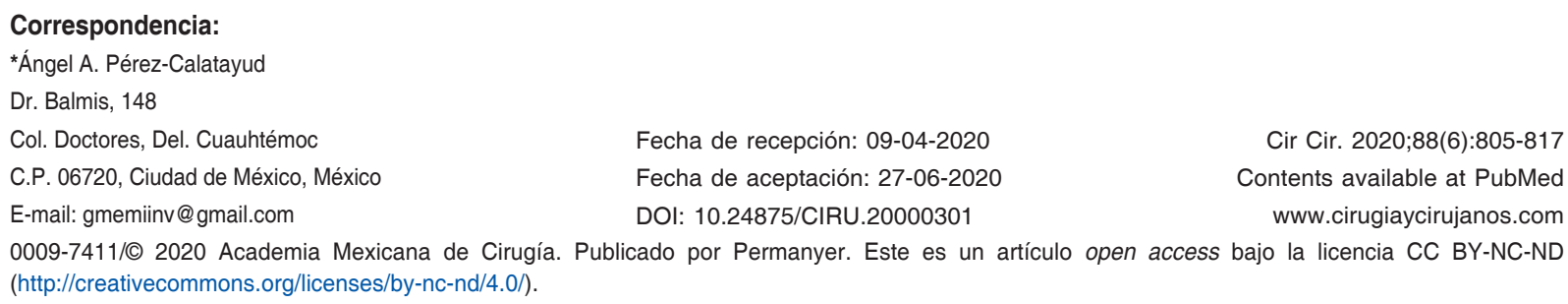

Cir Cir. 2020;88(6):805-817 Contents available at PubMed www.cirugiaycirujanos.com 0009-7411/@ 2020 Academia Mexicana de Cirugía. Publicado por Permanyer. Este es un artículo open access bajo la licencia CC BY-NC-ND (http://creativecommons.org/licenses/by-nc-nd/4.0/). 


\section{Introducción}

EI SARS-CoV-2 tiene una alta afinidad por el receptor de la enzima convertidora de angiotensina 2 en los humanos, la cual se expresa en los neumocitos tipo II en los pulmones. Los reportes de los diferentes países muestran que el pulmón es el objetivo más vulnerable, por su alta susceptibilidad a los virus inhalados. La distribución de la enzima convertidora de angiotensina 2 en otros órganos podría explicar la disfunción multiorgánica observada en pacientes críticos con COVID-19'.

En los pacientes que ameritan ingreso a la unidad de terapia intensiva, el soporte hemodinámico y ventilatorio es la piedras angular del tratamiento.

El objetivo de esta revision es dar a conocer las recomendaciones actuales sobre el soporte hemodinámico y ventilatorio en los pacientes con COVID-19 en fase crítica.

\section{Método}

La estrategia de búsqueda se realizó en PubMed y Trip Database. Debido a la escasez de publicaciones no se utilizó un algoritmo de búsqueda específico. Las publicaciones encontradas son estudios observacionales y recomendaciones de expertos; no hay metaanálisis ni estudios aleatorizados.

\section{Antecedentes}

Existen reportes epidemiológicos de la enfermedad grave del síndrome respiratorio agudo por el SARSCoV-2 donde existe un incremento en la morbilidad y la mortalidad en pacientes con enfermedad pulmonar crónica, tabaquismo, comorbilidad (hipertensión, diabetes 0 enfermedad cardiovascular) o edad avanza$\mathrm{da}^{2}$, y depende de la interacción de cuatro factores:

- Gravedad de la infección.

- Tiempo transcurrido desde el inicio de la enfermedad.

- El huésped (respuesta, reserva fisiológica y comorbilidad).

- Capacidad de respuesta ventilatoria del paciente a la hipoxemia ${ }^{3}$.

\section{Síndrome respiratorio agudo grave}

El síndrome de insuficiencia respiratoria aguda (SIRA) es un síndrome clínico definido por hipoxemia de inicio agudo (relación $\mathrm{PaO}_{2} / \mathrm{FiO}_{2}<300$ ) y opacidades pulmonares bilaterales en ausencia de insuficiencia cardiaca o por sobrecarga de volumen (aunque pueden coexistir) ${ }^{4}$.

La definición de Berlín estratifica el SIRA en tres subgrupos según el grado de hipoxemia (leve, moderada y grave $)^{5}$ de la siguiente forma:

- Leve: $\mathrm{PaO}_{2} / \mathrm{FiO}_{2}>200$ y $<300$ con uso de presión positiva al final de la espiración (PEEP) o presión positiva continua en la vía respiratoria (CPAP) $>5 \mathrm{cmH}_{2} \mathrm{O}$.

- Moderado: $\mathrm{PaO}_{2} / \mathrm{FiO}_{2}>100$ y $<200$ con uso de PEEP O CPAP $>5 \mathrm{cmH}_{2} \mathrm{O}$.

- Grave: $\mathrm{PaO}_{2} / \mathrm{FiO}_{2}<100$ con uso de PEEP o CPAP $>5 \mathrm{cmH}_{2} \mathrm{O}$.

La mortalidad incrementa a medida que disminuye la $\mathrm{PaO}_{2}: \mathrm{FiO}_{2}$ en pacientes con SIRA de moderado a grave (relación $\mathrm{PaO}_{2}: \mathrm{FiO}_{2}<150 \mathrm{mmHg}$ ).

Los pacientes con COVID-19 pueden desarrollar SIRA con características propias de esta infección viral. Los pulmones presentan una distensibilidad mayor que la observada en el SIRA. La hipoxia es grave y prevalece conforme se va perdiendo la regulación de la perfusión pulmonar, se produce una gran vasoconstricción hipóxica y los neumocitos con efecto citopático viral producen una hiperinflamación que continúa en etapas avanzadas, lo que resulta en un compromiso pulmonar grave ${ }^{6}$. Estos pacientes con hipoxemia grave ${ }^{7}$ manifiestan cierta uniformidad en su evolución: respiración normal, «hipoxemia silenciosa» o disnea, hipocapnia, normocapnia o hipercapnia, y en etapas graves pueden responder o no a la posición de prono; a pesar de los parámetros de ventilación mecánica protectora, la mortalidad dentro de las unidades de terapia intensiva es muy elevada ${ }^{8-10}$.

De acuerdo con la gravedad de los síntomas, los pacientes pueden ser clasificados como leves, graves y críticos (Tabla 1$)^{11}$.

Tabla 1. Gravedad de los síntomas. El espectro de la COVID-19 ha sido reportado en un rango que abarca desde infección asintomática hasta fallo respiratorio grave

\begin{tabular}{ll}
\hline Tipos clínicos & Síntomas \\
\hline Leve & Ausencia de neumonía o neumonía leve \\
Grave & $\begin{array}{l}\text { Disnea, frecuencia respiratoria } \geq 30, \mathrm{SO}_{2} \leq 93 \%, \\
\\
\mathrm{PaO}_{2} / \mathrm{FiO} \mathrm{O}_{2}<300, \text { o infiltrados pulmonares }>50 \% \\
\\
\text { dentro de } 24 / 48 \mathrm{~h}\end{array}$ \\
Crítico & Falla respiratoria, choque séptico o disfunción \\
& orgánica múltiple \\
\hline
\end{tabular}




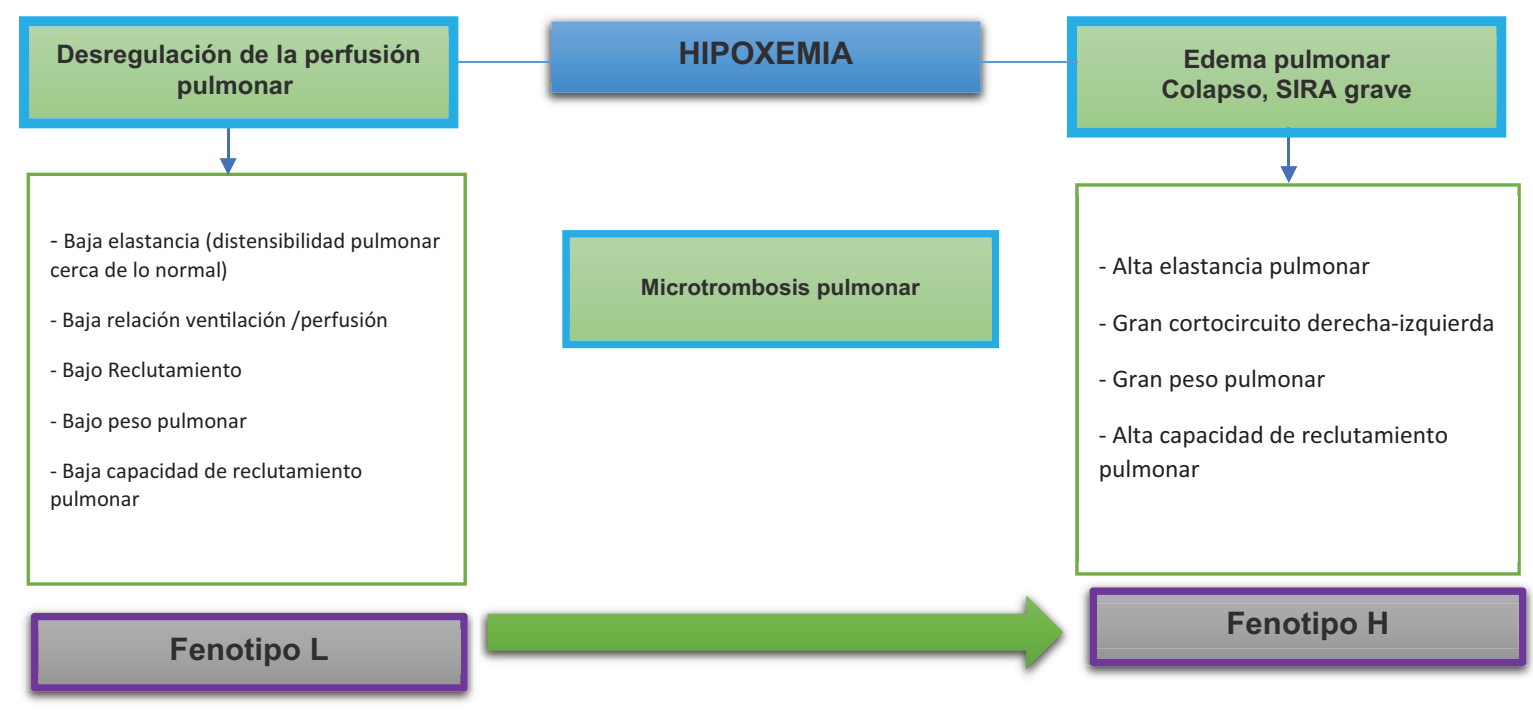

Figura 1. COVID-19, relación de los fenotipos $L y H^{69}$; Adaptado de Repessé $X$, et al..$^{53}$

\section{Fenotipos asociados (Fig. 1)}

Se sabe poco sobre la interacción genética del huésped con la infección y su progresión. Los datos relacionados con el sistema inmunitario y los modelos de predicción del riesgo genético se han relacionado con subtipos de células inmunitarias y hematológicas involucrados en la defensa viral, incluido el recuento de linfocitos.

Existe una transición entre fenotipos. Los pacientes con neumonía con fenotipo tipo $L$ pueden permanecer sin cambios durante un período y luego mejorar o presentar un deterioro de la funcion pulmonar, lo que determina la evolución y la gravedad de la enfermedad ${ }^{10}$.

\section{Fenotipo $L$}

Se caracteriza por una disminución de la relación entre ventilación y perfusión (V/Q). La presión de la arteria pulmonar no presenta cambios importantes, aunque se ha descrito cor pulmonale asociado en estos pacientes y existe pérdida de la regulación de la perfusión por vasoconstricción hipóxica.

Se pueden corroborar mediante tomografía computarizada (TC) la presencia de edema intersticial subpleural local y la imagen de vidrio esmerilado subpleural a lo largo de las cisuras pulmonares, que se relacionan con la baja capacidad de reclutamiento alveolar.

En etapas avanzadas, el paciente presenta hipoxemia grave que se asocia con un incremento negativo de la presión inspiratoria intratorácica, un incremento del volumen corriente en respiración espontánea y un incremento de la permeabilidad pulmonar debido a la gravedad del proceso inflamatorio con presencia de edema intersticial. Estas alteraciones son la principal causa de lesión pulmonar autoinfligida. En esta etapa se desarrollan disnea y deterioro de la función pulmonar, con aumento del trabajo ventilatorio. La transición del tipo $\mathrm{L}$ al tipo $\mathrm{H}$ puede deberse a la replicación viral y a la lesión pulmonar autoinfligida ${ }^{12}$.

\section{Fenotipo $\mathrm{H}$}

Puede presentarse en el $20-30 \%$ de los pacientes. Al ingreso presentan un grave edema pulmonar alveolar e intersticial con criterios graves de SIRA: hipoxemia, infiltrados bilaterales, compromiso de la elastancia pulmonar producido por la disminución del volumen de gas, incremento del cortocircuito de derecha a izquierda por el aumento de la fracción del gasto cardiaco que perfunde las áreas pulmonares no ventiladas y presenta una alta capacidad de reclutamiento pulmonar.

El daño directo del virus produce una lesión hiperinflamatoria, compromiso pulmonar grave, daño alveolar difuso y una gran tormenta de citocinas, y se ha descrito en la TC pulmonar un incremento notable en el peso del pulmón $(>1.5 \mathrm{~kg})^{6,13}$.

\section{Etapas de la inflamación}

\section{Etapa I: fase viral}

El recuento de glóbulos blancos tiende a ser normal, la linfocitopenia se observa en el $80 \%$ de los 
pacientes, y existe trombocitopenia leve; ambos reconocidos como un signo de mal pronóstico y evoluciona a una coagulación intravascular diseminada de tipo trombótico, la cual afecta el centro lobulillar de los alveolos e incrementa las alteraciones V/Q observadas en los pacientes críticos. Los marcadores inflamatorios, como la proteína $C$ reactiva, se encuentran incrementados y en esta fase ayuda al diagnóstico diferencial con otras patologías que cursan con insuficiencia respiratoria grave y proteína $C$ reactiva normal. Los valores elevados de ferritina sérica ayudan a la detección de un síndrome de tormenta de citocinas en los pacientes con fiebre alta ${ }^{14}$.

\section{Etapa II: fase pulmonar}

A pesar de estar estable durante varios días durante la etapa I, el paciente puede deteriorarse abruptamente y presentar insuficiencia respiratoria hipoxémica. Los marcadores de inflamación sistémica a menudo son moderadamente elevados (proteína C reactiva y ferritina). Se produce una respuesta inmunitaria adaptativa, que provoca una reducción en los títulos virales. Sin embargo, esto también conduce a mayor inflamación y más daño tisular ${ }^{15,16}$.

\section{Etapa III: fase de hiperinflamación}

Los pacientes se deterioran de manera progresiva, presentan coagulación intravascular diseminada y síndrome de falla multiorgánica. Las anormalidades de laboratorio incluyen el incremento del dímero $D$ (> 1500 asociado a mal pronóstico), de la proteína C reactiva y de la ferritina. Inicialmente, los pacientes pueden responder bien a la intubación y la ventilación durante la etapa II, pero posteriormente la grave producción de citocinas y la activación endotelial con la consecuente activación de la coagulación conducen a un deterioro clínico. La respuesta inmunitaria adaptativa se convierte en una tormenta de citocinas inmunopatológicas desreguladas. Esto probablemente representa una forma de síndrome hiperferritinémico, lo cual debe diferenciarse si se trata de un síndrome de activación del macrófago o un antifosfolípido catastrófico ${ }^{17}$. La microtrombosis y los eventos isquémicos asociados también son comunes (incluyendo accidentes cerebrovasculares). Los niveles de dímero $D$, proteína $C$ reactiva y ferritina incrementan conforme a la gravedad y se asocian a una mayor mortalidad ${ }^{18}$.
En observaciones en autopsias realizadas en el Hospital de Sacco, en Italia, en la disertación anatomopatológica, histológicamente el pulmón presenta áreas muy enfisematosas con vasos sanguíneos dilatados. Se observa gran daño alveolar difuso con formación de membranas hialinas y gran exudado fibrótico. La diferencia del SIRA con la COVID-19 es que durate el monitoreo de la ventilacion su comportamiento es similar al del enfisema, y existe formación de microtrombos vasculares y microhemorragias, con pequeñas zonas de daño alveolar difuso ${ }^{19,20}$.

\section{Ventilación mecánica invasiva (Fig. 2)}

Existe una relación entre la neumonía viral y una mayor predisposición a una lesión pulmonar autoinfligida inducida por el ventilador, debido a que la ventilación mecánica somete al pulmón a fuerzas que alteran su estructura y sus propiedades elásticas. En pulmones lesionados por COVID-19, el patrón de inflación es heterogéneo y la fuerza de distensión alveolar puede alcanzar hasta $140 \mathrm{cmH}_{2} \mathrm{O}$, aun con presiones transpulmonares de $30 \mathrm{cmH}_{2} \mathrm{O}$, siendo susceptibles al daño cíclico por fuerzas de cizallamiento resultantes del colapso y la sobredistensión repetitivos, afectando a todos los componentes estructurales y celulares del pulmón que contribuyen a una lesión ocasionada por el ventilador. Al incrementar la presión alveolar durante la inflación se disminuye la presión intersticial y se incrementa la presión transmural de los vasos adyacentes; sin embargo, en los vasos intraalveolares ocurre lo contrario, pues con el incremento del volumen pulmonar se comprimen y hay cese circulatorio, modificando la resistencia vascular pulmonar y contribuyendo a la heterogeneidad. El incremento de la permeabilidad vascular puede llevar a la formación de gran edema alveolar, comprometiendo el intercambio gaseoso. La hipoxemia debe tratarse en primera instancia con $\mathrm{FiO}_{2}$ alta, sin apresurarse a alcanzar objetivos a corto plazo; cualquier intervención puede ser más perjudicial que ganar tiempo (Fig. 2) 21,22.

- Volumen corriente de 4-6 $\mathrm{ml} / \mathrm{kg}$ de peso predicho.

- Peso predicho: mujeres, (talla en $\mathrm{cm}-152.4) \times$ $0.91+45.5$; hombres, (talla en $\mathrm{cm}-152.4) \times 0.91$ +50 .

- La presión plateau se debe mantener por debajo de $30 \mathrm{cmH}_{2} \mathrm{O}$.

- Meta de oxigenación del 88-92\% ${ }^{23,24}$.

La PEEP debe limitarse a 8-10 $\mathrm{cmH}_{2} \mathrm{O}$, ya que niveles más altos no incrementarán la presión pulmonar e 


\section{DETERIORO}
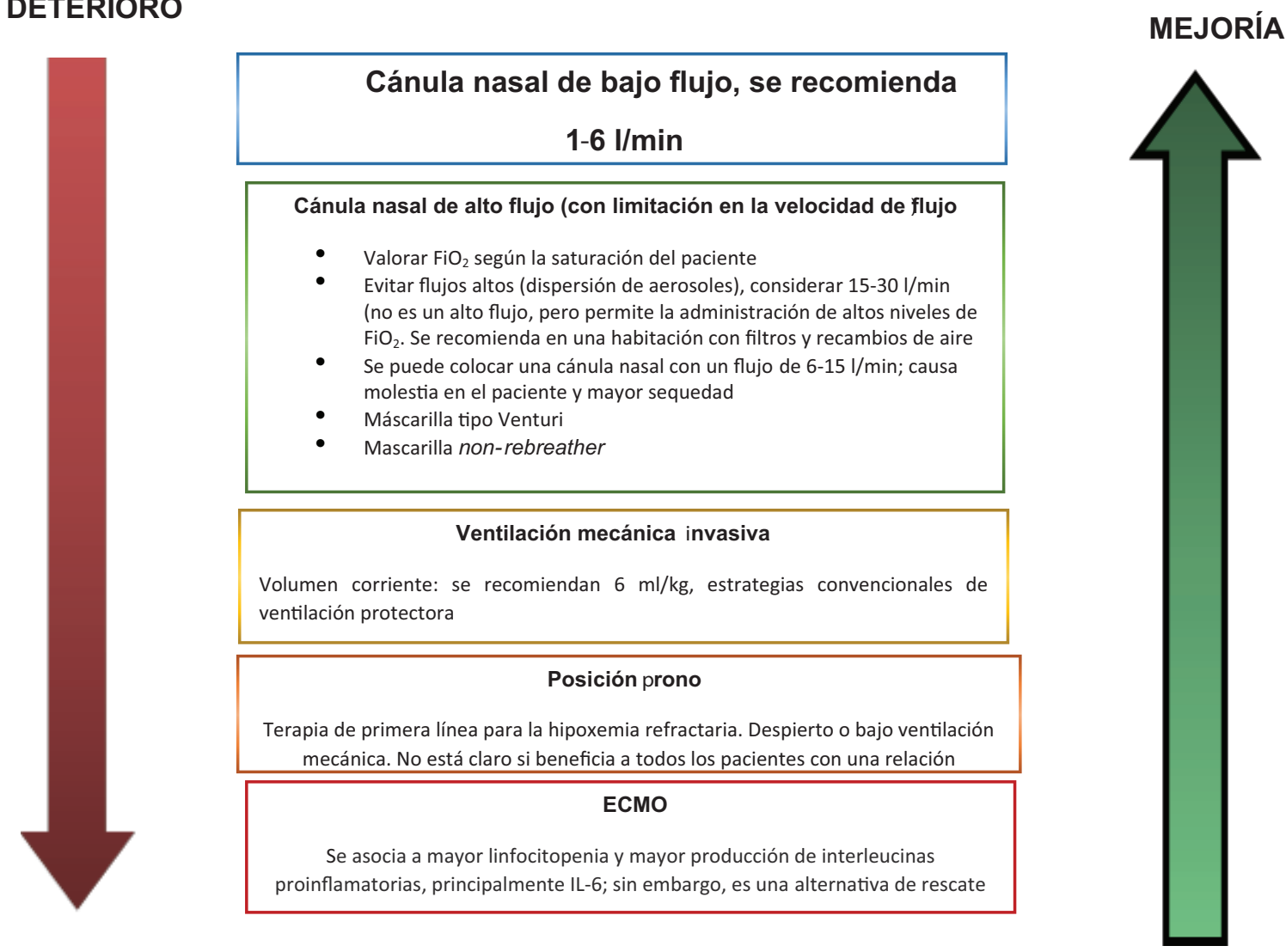

Figura 2. Esquema general de soporte ventilatorio en pacientes con COVID-19. Adaptado de Marik P. ${ }^{21}$

impactarán en el retorno venoso con un daño mayor que el beneficio esperado. Se recomienda que los niveles de PEEP se mantengan bajos, los umbrales de volumen corriente no deben limitarse a $6 \mathrm{ml} / \mathrm{kg}$, en pacientes con gran disoxia, se puede considerar volúmenes superiores a $6 \mathrm{ml} / \mathrm{kg}$, y la frecuencia respiratoria no debe exceder las 20 respiraciones por minuto ${ }^{25}$ El valor de driving pressure (presión plateau-PEEP) se debe manejar $<15 \mathrm{cmH}_{2} \mathrm{O}$ (normalmente los pacientes no son respondedores a PEEP), lo que se ha asociado a una menor mortalidad. Un nivel de $\mathrm{PaO}_{2}>50 \mathrm{mmHg}$ sugiere atelectasia por hipoventilación $n^{4,26}$

\section{Maniobras de reclutamiento}

Tienen poco valor, pero se pueden aplicar presiones moderadas de aproximadamente $30 \mathrm{cmH}_{2} \mathrm{O}$ durante 20-30 segundos; si el paciente no presenta mejoría en la oxigenación o la presión de conducción, barotrauma o inestabilidad hemodinámica, las maniobras de reclutamiento deben interrumpirse. En cuanto a la TC, se debe realizar (considerando el alto riesgo de contaminación) con el objetivo de identificar el parénquima pulmonar reclutable. Esta fracción debe evaluarse con dos TC consecutivas en la misma sesión, con PEEP a niveles de 5 y $45 \mathrm{cmH}_{2} \mathrm{O}$, para medir la diferencia en la cantidad de ventilación del parénquima y el potencial de reclutamiento ${ }^{25}$.

\section{Retiro de la ventilación mecánica}

No debe acelerarse el retiro de la ventilación mecánica. De acuerdo con la experiencia actual de los centros especializados en China e Italia, este puede retrasarse hasta 2 semanas por la lenta evolución de los pacientes ${ }^{27}$. El retiro debe ser gradual, con despresurización lenta del sistema ventilatorio. Esto puede hacerse con una reducción gradual en el nivel de PEEP verificando que las metas de oxigenación se cumplan; un retiro precoz puede generar mayor deterioro y empeorar el pronóstico de los pacientes. Sin duda, la ventilación mecánica es necesaria como 
soporte vital de estos pacientes, pero debemos limitar su daño y utilizar el soporte mínimo que permita alcanzar la meta de oxigenación ${ }^{28}$.

\section{Oxigenación con membrana extracorpórea (ECMO)}

Es limitado su uso en pacientes con COVID-19. Se asocia a mayor linfocitopenia y mayor producción de interleucinas proinflamatorias, principalmente interleucina 6), por lo cual los pacientes con COVID-19 presentan estas dos características principalmente. En el ensayo EOLIA, el uso temprano de ECMO no mejoró significativamente la mortalidad a los 60 días en pacientes con SIRA grave ${ }^{29}$; sin embargo, se usa como modalidad de rescate en casos extremos ${ }^{30}$.

\section{Otras medidas de soporte}

El oxígeno nasal con cánula de alto flujo y la ventilación no invasiva solo deben usarse en pacientes seleccionados con insuficiencia respiratoria hipoxémica, y que se encuentren estables hemodinámicamente; deben ser monitorizados con estrecha vigilancia en la unidad de cuidados críticos, y si el paciente no responde en 1 hora y se deteriora agudamente no se debe retrasar la intubación orotraqueal y asistir con ventilación mecánica invasiva ${ }^{30,31}$.

En comparación con la oxigenoterapia estándar, el oxígeno nasal con cánula de alto flujo reduce la necesidad de intubación, pero se debe considerar que existe mayor dispersión de aerosoles y se asocia a mayor contaminación, por lo que es fundamental que se realice en una habitación con filtros y con recambios de aire. En pacientes infectados con coronavirus, los estudios son limitados y poco recomendables.

Las guías de ventilación no invasiva no recomiendan su uso en la insuficiencia respiratoria hipoxémica; sugieren una alta tasa de fracaso, retrasan el soporte ventilatorio mayor inestabilidad hemodinámica, y los pacientes en respiración espontánea o semiasistida (CPAP/ventilación no invasiva) durante mucho tiempo tienen una presión intratorácica altamente negativa, alta distensibilidad pulmonar y deterioro aun con medidas de soporte ventilatorio y hemodinámico, debido a que se produce lesión pulmonar inducida por ventilación y superinfección bacteriana. En estos casos, puede ser más cauteloso incrementar gradualmente la PEEP. Se recomienda el monitoreo de la $\mathrm{ScO}_{2}$, y considerar cuando la $\mathrm{SvO}_{2}$ comienza a disminuir, el gasto cardiaco se encuentra deteriorado y los niveles de PEEP ya no son útiles para el reclutamiento pulmonar; es por eso que se debe evaluar la función cardiaca derecha mientras se manejan los incrementos de PEEP ${ }^{32}$.

\section{Uso de ventilador dual}

Ante una medida de emergencia y de situaciones de desastre, con sobresaturación de los hospitales, pero no es recomendable usarlo debido a varias condiciones. Si todos los pacientes conectados a un solo ventilador tienen las mismas características clínicas al inicio, podrían deteriorarse y recuperarse a diferentes velocidades, y la distribución de gas a cada paciente sería desigual; por lo tanto, en el escenario del paciente más grave obtendría el volumen corriente más pequeño y el paciente que mejora obtendría el volumen corriente más grande. La mecánica pulmonar sería difícil de medir, incrementando así los errores en la medición. La PEEP ${ }^{33}$, que es de importancia crítica en estos pacientes, sería imposible de manejar; los volúmenes irían a los segmentos pulmonares más distensibles. En caso de un paro cardiaco sería necesario detener la ventilación de todos los pacientes. Al momento del cambio a la ventilación, por la dispersión de aerosol viral se expondría a los trabajadores de la salud y demás pacientes. Por lo tanto, no es un opción viable, de acuerdo con las recomendaciones de la Association for Respiratory Care, la American Society of Critical Care Medicine y el American College of Chest Physicians ${ }^{34}$.

\section{Posición de decúbito prono}

Debe considerarse como una maniobra de rescate para facilitar la redistribución de flujo sanguíneo pulmonar, en lugar de abrir áreas colapsadas. Cuando a pesar de administrar altas concentraciones de oxígeno la $\mathrm{PaO}_{2} / \mathrm{FiO}_{2}$ es $<150$, es necesario colocar al paciente en decúbito prono lo antes posible y ventilar en esta posición al menos 16 horas. Los balances hídricos deberán ser negativos para evitar un exceso de líquidos en el pulmón ${ }^{35}$. El importante aumento del peso pulmonar puede causar compresión de las regiones pulmonares dorsales al $50 \%$ de la altura del pulmón. Debido a las fuerzas de compresión relacionadas con el peso cuando el paciente se vuelve propenso, el pulmón se colapsa al $50 \%$ de su altura (la región ventral), mientras que las regiones por encima del $50 \%$ de la altura (las regiones dorsales) se 
volverán a abrir. El efecto sobre el intercambio de gases dependerá de la cantidad de masa presente en el $50 \%$ superior y el $50 \%$ inferior.

\section{Posición de prono}

En este momento hay poca información sobre la forma ideal de manejar a los pacientes con SIRA asociado al SARS-CoV-2, y sobre el papel específico de el síndrome de insuficiencia respiratoria aguda (DP) en esos pacientes. Sin embargo, los primeros informes chinos describen la mecánica respiratoria y la capacidad de reclutamiento pulmonar en una pequeña cohorte de pacientes con ventilación mecánica con SIRA asociado al SARS-CoV-2. Los principales hallazgos fueron que ninguno de los pacientes incluidos tenía cierre completo de las vías aéreas ni auto-PEEP, y la presión de conducción fue alta (en promedio $22 \mathrm{cmH}_{2} \mathrm{O}$ a pesar de usar $6 \mathrm{ml} / \mathrm{kg}$ de volumen corriente). Utilizaron un índice para cuantificar directamente el potencial de reclutamiento pulmonar, llamado relación reclutamiento-inflación (relación R/l), que estima cuánto incremento en el volumen pulmonar espiratorio final inducido por PEEP se distribuye entre el pulmón reclutado (reclutamiento) y la inflación y/o hiperinflación del «pulmón bebé» cuando se aplica una PEEP más alta. La mayoría de los pacientes incluidos eran poco reclutables con PEEP alta, pero la capacidad de reclutamiento parecía cambiar cuando se alternaba la posición del cuerpo. En los pacientes en posición prona, la $\mathrm{PaO}_{2} / \mathrm{FiO}_{2}$ pasó de $120 \pm 61$ $\mathrm{mmHg}$ en supino a $182 \pm 140 \mathrm{mmHg}$ en prono $(p=$ 0.065 por prueba $t$ student) ${ }^{36}$. Sun, et al..$^{37}$ también describieron la aplicación de DP en pacientes despiertos como un tratamiento temprano de cuidados críticos en pacientes de alto riesgo (frecuencia cardiaca $>120$ o frecuencia respiratoria $>30,0 \mathrm{SpO}_{2}<$ $93 \%$ de aire en la sala de respiración) con alta sospecha o neumonía asociada al SARS-CoV-2 confirmada. EI DP despierto, junto con la restricción de líquidos y el uso de oxigenoterapia de cánula nasal de alto flujo o ventilación mecánica no invasiva para conservar la PEEP, les permite mantener la tasa de ventilación mecánica invasiva por debajo del 1\%, que fue significativamente bajo, en especial en esta población específica. Todavía hay falta de información sobre la mejor estrategia y el tiempo de implementación de DP en pacientes despiertos, así como la duración ideal y la mejor manera de evaluar el éxito o el fracaso para evitar retrasos en la implementación de la ventilación mecánica invasiva ${ }^{37}$.

\section{Posición de prono despierto}

Los beneficios de la DP en pacientes con ventilación mecánica están bien establecidos; sin embargo, algunos autores han aplicado la DP en pacientes con ventilación no invasiva y despiertos con resultados prometedores. Scaravilli, et al. ${ }^{38}$ abordaron los beneficios de la DP en 15 pacientes con alto riesgo de adquirir neumonía asociada al ventilador si se estableciera la ventilación mecánica invasiva. Los pacientes fueron manejados con diferentes dispositivos, PEEP y niveles de $\mathrm{FiO}_{2}$. La mediana de duración de los ciclos de DP fue de 3 horas (2-4) y el procedimiento más largo duró 8 horas. Observaron una mejoría en la oxigenación en todos los pacientes, pero volvieron a los valores basales 6 horas después de la resupinación, siendo necesarios varios ciclos de DP. Los parámetros hemodinámicos y la frecuencia respiratoria no afectaron durante la DP. La tasa de supervivencia fue del $80 \%$ y la necesidad de ventilación mecánica invasiva fue del $13 \%{ }^{39}$.

Gattinoni, et al. ${ }^{40}$ han emitido una observación preliminar acerca de la lesión pulmonar producida por este virus, en la cual destacan dos escenarios clínicos en su experiencia durante la epidemia en Lombardía, Italia. En los pacientes con distensibilidad pulmonar alta y neumonía viral aislada el hallazgo principal es la presencia de vasoconstricción hipóxica explicando la hipoxemia grave. En estos pacientes, el mayor problema se relaciona con la perfusión, los pulmones se encuentran dilatados y el incremento en la PEEP no confiere beneficios. La PEEP alta y la posición de prono no conducen al reclutamiento de áreas colapsadas, pero pueden ser benéficas para optimizar la perfusión pulmonar. Los patrones tomográficos en este escenario clínico muestran que no hay áreas potencialmente reclutables en forma significativa y la presencia de cortocircuitos mayores del $50 \%$.

\section{Manejo hemodinámico}

Los pacientes con COVID-19 pueden desarrollar SIRA y evolucionan a cuatro momentos críticos a nivel hemodinámico. En caso de hipotensión se recomienda un reto de vasopresores, basándose en un principio fisiológico:

- El primero de ellos es una vasoconstricción pulmonar hipóxica que puede ocasionar hipertensión pulmonar y a su vez disfunción del ventrículo derecho. Estas dos situaciones pueden hacer que 
el paciente sea muy poco tolerante a cargas de volumen intravascular, lo que se puede evidenciar fácilmente por ultrasonido al encontrar un ventrículo derecho del mismo tamaño o mayor que el ventrículo izquierdo, junto con una vena cava inferior dilatada y sin colapso ${ }^{41}$.

- El segundo tipo de crisis hemodinámica de estos pacientes es cuando se encuentran en la llamada "tormenta de citocinas»" terizada por niveles de interleucina 6 altos, proteína $C$ reactiva $>100 \mathrm{mg} / \mathrm{dl}$, ferritina $>600 \mu \mathrm{g}$ y eventos de fiebre $>38^{\circ} \mathrm{C}$. Esto puede causar un estado de sepsis, vasodilatación o depresión miocárdica, incluyendo la rotura de placas ateromatosas en las coronarias que lleven a un infarto agudo del miocardio.

- El tercero son las crisis relacionadas con eventos trombóticos, como la tromboembolia pulmonar, que se ha asociado a niveles de dímero $\mathrm{D}$ altos al ingreso de estos pacientes ${ }^{43,44}$.

- El cuarto y más grave evento de crisis hemodinámica de estos pacientes es un cuadro de choque cardiogénico causado por una miocarditis fulminante viral ${ }^{45}$.

Por lo tanto, se recomienda no hacer ninguna prueba estática o dinámica de respuesta a volumen por dos motivos. En primer lugar, los pacientes con algún grado de hipertensión pulmonar difícilmente son respondedores a volumen. En segundo lugar, y más importante, todas las pruebas dinámicas o estáticas de respondedor a volumen carecen de validez fisiológica ${ }^{46}$ en escenarios de hipertensión pulmonar. De hecho, la variabilidad de la presión de pulso puede verse incrementada como dato de disfunción de ventrículo derecho y no como dato de respuesta a volumen ${ }^{47}$.

Cuando el paciente no se encuentre en un evento de tormenta de citocinas, la meta es lograr balances negativos. Se recomienda el protocolo FACTT (Fluids and Catheters Treatment Trial) simplificado de restricción de volumen y el empleo de un diurético. El protocolo FACTT simplificado ${ }^{48}$ consiste en:

- Descontinuar los fluidos de mantenimiento.

- Dilución de medicamentos en la menor cantidad compatible posible.

- No limitar los requerimientos de nutrición enteral.

- Utilizar vasopresores en caso de presión arterial media $<60 \mathrm{mmHg}$.

Ante una creatinina sérica $\geq 3 \mathrm{mg} / \mathrm{dl}$ durante el protocolo se evaluará la necesidad de hemodiálisis.
Se recomienda usar el reto de vasopresor antes que el reto de volumen para revertir eventos de hipotensión.

No se recomienda la realización de pruebas dinámicas o estáticas para evaluar la respuesta a fluidos.

Recomendamos el protocolo FACTT simplificado para el manejo de volumen en pacientes con COVID-19 que no se encuentren en tormenta de citocinas.

Los pacientes con COVID-19 en crisis de tormenta de citocinas pueden ser ubicados en el fenotipo inflamatorio de SIRA ${ }^{49}$. Se ha descrito que este grupo no se beneficia de la restricción de volumen. Por el tiempo de duración de la fiebre se estima el déficit de volumen ${ }^{50}$ y se inicia un bolo de solución Ringer lactato de $250 \mathrm{ml}$, al mismo tiempo que norepinefrina. De manera empírica recomendamos un reto de volumen solo mientras se tenga una presión venosa central < $8 \mathrm{mmHg}$.

\section{Recomendaciones hemodinámicas}

La norepinefrina ${ }^{51}$ puede redistribuir el flujo pulmonar, lo que disminuiría la hipertensión pulmonar, que es una de las causas de hipotensión de estos pacientes, pero dosis altas de norepinefrina pueden exacerbar la hipertensión pulmonar, por lo que recomendamos, antes de usar dosis altas de norepinefrina, agregar vasopresina ${ }^{52}$, que tiene una menor repercusión en la presión arterial pulmonar.

Por otro lado, desde el punto de vista hemodinámico recomendamos el empleo de fármacos de inducción con mínimos efectos hemodinámicos, que pueden consultarse en otros escritos al respecto.

Es importante en lo posible contar con una evaluación ecocardiográfica. El manejo hemodinámico de estos pacientes se determina adecuado si se reduce el grado de hipertensión pulmonar o disminuye el tamaño del ventrículo derecho como respuesta a cada intervención. La presencia de cor pulmonale (hipertensión pulmonar) puede sospecharse mediante una escala diseñada por el grupo de trabajo de VieiIlard-Baron ${ }^{27,53}$. La presencia de más de 2 puntos en la escala de cor pulmonale (Tabla 2) debe advertir de algún grado de hipertensión pulmonar, y este es el grupo de mayor mortalidad en pacientes con SIRA. En estos casos debe mantenerse el equilibrio de $\mathrm{PaCO}_{2}<48 \mathrm{mmHg}$ junto con una presión meseta $<27 \mathrm{cmH}_{2} \mathrm{O}$ y una relación meseta-PEEP $<18 \mathrm{cmH}_{2} \mathrm{O}$. Lo anterior debe considerarse como parte de la 
Tabla 2. Escala pronostica de cor pulmonale ${ }^{15}$

\begin{tabular}{lc}
\hline Parámetro & Puntos \\
\hline Neumonía como causa de SIRA & 1 \\
Meseta $-\mathrm{PEEP}>18 \mathrm{cmH}_{2} \mathrm{O}$ & 1 \\
$\mathrm{PaO}_{2} / \mathrm{FiO}_{2}<150$ & 1 \\
$\mathrm{PaCO}_{2}>48 \mathrm{mmHg}$ & 1 \\
Total & $0-4$ \\
\hline
\end{tabular}

estrategia hemodinámica de protección del ventrículo derecho ${ }^{27}$.

\section{Sospecha de hipoperfusión}

Criterios clínicos:

- Escala de piel marmórea $>2^{54}$.

- Llenado capilar $^{55}>4$ segundos en rodillas y $>2.5$ segundos en dedo índice.

- Eventos de hipotermia $<36^{\circ} \mathrm{C}$.

- Índice de choque (frecuencia cardiaca / presión arterial sistólica $>1$ ).

- Frecuencia cardiaca $<60$ + presión arterial sistólica $<90 \mathrm{mmHg}^{56}$.

Criterios gasométricos de bajo gasto:

- $\mathrm{SvcO}_{2}<65 \%$.

- Gap ${ }^{57}$ venoarterial de $\mathrm{CO}_{2}=$ presión venosa de $\mathrm{CO}_{2}(\mathrm{PvCO} 2)$ - presión arterial de $\mathrm{CO}_{2}\left(\mathrm{PacO}_{2}\right)$ $>6 \mathrm{mmHg}$.

Ante un criterio clínico más un criterio gasométrico, o tres criterios clínicos, o los dos gasométricos, la probabilidad de hipoperfusión es muy alta. Se recomienda utilizar el algoritmo como una guía de abordaje. En caso de que el monitoreo ecocardiográfico o mínimamente invasivo no sea suficiente, y en especial en los casos en que se considere el uso de inodilatadores de arteria pulmonar, sugerimos el monitoreo mediante catéter de arteria pulmonar.

\section{Recomendaciones del manejo hemodinámico}

- Ante eventos de tormenta de citocinas recomendamos no seguir estrategias de restricción de líquidos, pero regresar a estas una vez que se controle el evento.

- Evaluar el empleo de norepinefrina y vasopresina antes que dosis altas de norepinefrina en el manejo de la hipotensión asociada a crisis de vasoconstricción pulmonar hipóxica.

- El reto de inotrópico, de continuar el deterioro hemodinámico con $\mathrm{SvCO} 2<60 \%$ o Gap vaCO 2 $>6 \mathrm{mmHg}$, así como seguimiento por parámetros de $\mathrm{SvcO}_{2}$ y $\mathrm{Gap} \mathrm{vaCO}_{2}$ para evaluar la respuesta terapéutica.

- Recomendamos, en casos que persistan con deterioro hemodinámico atribuible a hipertensión pulmonar, evaluar terapias de vasodilatación pulmonar como óxido nítrico, iloprost, etc., y considerar el uso de monitoreo avanzado invasivo de la presión de la arteria pulmonar.

\section{Monitorización ultrasonográfica de la ventilación mecánica}

El ultrasonido pulmonar es una herramienta dinámica, de bajo costo, no invasiva y a la cabecera del enfermo, que permite evaluar las intervenciones realizadas, ya sean estrategias ventilatorias 0 incluso farmacológicas, pero requiere complementación con otras modalidades de monitorización, dentro de las cuales en forma obligada se incluye la valoración de la función ventricular derecha buscando en forma intencionada datos de sobredistención.

La monitorización de la ventilación mecánica con ultrasonido a la cabecera del enfermo confiere varias ventajas:

- Es una estrategia no invasiva.

- Evalúa el estado del parénquima pulmonar y la respuesta a intervenciones terapéuticas, tanto estrategias ventilatorias ${ }^{58}$ como la tendencia evolutiva ante la administración de medicamentos.

- Permite evaluar la función cardiaca ${ }^{59}$.

- Facilita la categorización de pacientes potencialmente complicables de manera oportuna ${ }^{60}$.

\section{Titulación de la PEEP guiada por ultrasonografía pulmonar}

La ventilación mecánica con volúmenes corrientes bajos y PEEP alta es una estrategia convencional en pacientes con SIRA para mejorar la oxigenación ${ }^{61}$. Sin embargo, existen casos en los cuales este objetivo no es alcanzado y se requieren maniobras de reclutamiento alveolar para su adecuada apertura, mismas que se deben monitorizar estrechamente ya que pueden conducir a inestabilidad hemodinámica ${ }^{62}$.

Dentro de estos estudios se ha sugerido el uso del índice de aireación para determinar la mejor PEEP en 
Tabla 3. Índice de aeración por ultrasonido pulmonar

\begin{tabular}{|c|c|c|c|c|c|}
\hline \multicolumn{3}{|c|}{ Cuantificación de reaeración } & \multicolumn{3}{|c|}{$\begin{array}{c}\text { Cuantificación de pérdida de } \\
\text { reaeración }\end{array}$} \\
\hline $\begin{array}{l}+1 \\
\text { punto }\end{array}$ & $\begin{array}{l}+3 \\
\text { puntos }\end{array}$ & $\begin{array}{l}+5 \\
\text { puntos }\end{array}$ & -5 puntos & -3 puntos & -1 punto \\
\hline $\mathrm{B} 1 \rightarrow \mathrm{N}$ & $\mathrm{B} 2 \rightarrow \mathrm{N}$ & $\mathrm{C} \rightarrow \mathrm{N}$ & $\mathrm{N} \rightarrow \mathrm{C}$ & $\mathrm{N} \rightarrow \mathrm{B} 2$ & $\mathrm{~N} \rightarrow \mathrm{B} 1$ \\
\hline $\mathrm{B} 2 \rightarrow \mathrm{B} 1$ & $\mathrm{C} \rightarrow \mathrm{B} 1$ & & & $\mathrm{~B} 1 \rightarrow \mathrm{C}$ & $\mathrm{B} 1 \rightarrow \mathrm{B} 2$ \\
\hline $\mathrm{C} \rightarrow \mathrm{B} 2$ & & & & & $\mathrm{~B} 2 \rightarrow \mathrm{C}$ \\
\hline
\end{tabular}

Monitorización ecocardiográfica de la ventilación mecánica.

diferentes segmentos pulmonares, dividiendo en cuatro segmentos cada hemitórax y reconociendo cuatro patrones:

- N: patrón de ultrasonido pulmonar normal.

- B1: múltiples líneas B con un espacio de $7 \mathrm{~mm}$ entre ellas (pérdida moderada de la aireación).

- B2: múltiples líneas B coalescentes (pérdida grave de la aireación).

- C: consolidación pulmonar.

Una vez reconocidos los patrones pulmonares en los diferentes segmentos se puede otorgar mediante el índice de aireación por índice de pérdida de aireación un valor numérico (Tabla 3) ${ }^{63}$.

En pacientes con SIRA con ventilación mecánica se ha observado que el incremento de la PEEP por arriba de $10 \mathrm{cmH}_{2} \mathrm{O}$ se asocia a una disminución progresiva del gasto cardiaco y de la presión arterial media, lo que se ha relacionado con sobrecarga del ventrículo derecho y desplazamiento del tabique interventricular. Sin embargo, este análisis y otros semejantes fueron realizados cuando aún no se conocía la importancia clínica de la reducción del volumen corriente y su efecto sobre la apertura alveolar, lo que favorecía una ventilación con volúmenes corriente superiores a $10 \mathrm{ml} / \mathrm{kg}$ de peso ideal, lo que sin duda en pacientes con SIRA favorece la sobredistensión alveolar y la lesión pulmonar inducida por ventilación. Con base en el conocimiento adquirido, se sabe que la PEEP elevada genera incremento de la poscarga del ventrículo derecho, dilatación del ventrículo derecho, desplazamiento del tabique interventricular a la izquierda y disminución del volumen de eyección del mismo. La medición invasiva de la presión de los ventrículos derecho e izquierdo muestra que este efecto se relaciona con el gradiente de presión invertida entre los dos ventrículos. Los cambios en la función del ventrículo derecho relacionados con diferentes niveles de PEEP podrían reflejar el equilibrio entre reclutamiento y sobredistensión, de modo que si el incremento de la PEEP induce apertura alveolar hay disminución de la presión transpulmonar por incremento de la distenbilidad pulmonar y no existe involucro del ventrículo derecho. Sin embargo, si el incremento de la PEEP es excesivo existe sobredistensión alveolar y se produce elevación de la presión transpulmonar con incremento de la poscarga del ventrículo derecho ${ }^{64}$.

\section{Valoración del ventrículo derecho}

El papel de la ecocardiografía crítica no es solo de vigilancia diagnóstica, sino también de respuesta terapéutica.

Ante estas posibilidades, la monitorización de la ventilación mecánica en pacientes con COVID-19 se debe estar trabajando en conjunto con la unidad cardiopulmonar, ya que el $25 \%$ de los pacientes con SIRA por COVID-19 tienen una relación con la presencia de falla ventricular aguda derecha, y más del $60 \%$ de los pacientes con SIRA presentan falla hemodinámica respecto del monitoreo hemodinámico por ecocardiografía y la presentación de falla aguda del ventrículo derecho. Dentro de los parámetros de ecocardiografía crítica, en relación con la presentación de falla ventricular derecha aguda observamos cambios en la relación ventrículo derecho/ventrículo izquierdo basal, las resistencias vasculares pulmonares, la interdependencia ventricular para decidir el tipo de soporte del ventrículo derecho con inotrópicos, vasopresores o volumen según los hallazgos del monitoreo hemodinámico ecocardiográfico ${ }^{65}$.

\section{Presión sistólica pulmonar en el SIRA y falla ventricular aguda derecha}

Podemos hablar de la gravedad de la hipertensión pulmonar generada en el SIRA en relación a la presión sistólica de la arteria pulmonar, determinada por la distensibilidad de la vena cava inferior. Es importante recordar que la sincronía corazón-pulmón durante la ventilación mecánica con presión positiva u otros métodos, como airway pressure release ventilation (ARPV) ó ventilación con liberación de la presión de la vía aérea, altera el retorno venoso, por lo cual se pierde sensibilidad del cálculo de la presión de la aurícula derecha a través de la vena cava. En el $92 \%$ de los pacientes con SIRA existe algún grado de hipertensión pulmonar ${ }^{65}$. El valor de la presión sistólica pulmonar no tiene correlación directa con el desarrollo de falla ventricular aguda derecha. 
Al evaluar la falla ventricular derecha aguda, en términos de sobrevida, el mejor marcador pronóstico y diagnóstico de función sistólica del ventrículo derecho es el desplazamiento del anillo tricúspide. Otros parámetros, como la fracción de acortamiento, no se correlacionaron de manera adecuada. Los pacientes con SIRA por incremento de la poscarga comienzan a presentar sobrecarga volumétrica y vasopresora en el ventrículo derecho, lo que conlleva un desplazamiento sistólico del tabique interventricular hacia las cavidades izquierdas, con lo cual se pierde la contribución contráctil interventricular en el gasto cardiaco derecho, siendo este desplazamiento una de las manifestaciones más importantes en llevar al paciente a la falla cardiaca aguda derecha.

La valoración del desplazamiento del tabique en modo bidimensional puede ser difícil cuando no es muy marcado, por lo que la utilización del modo $\mathrm{M}$ facilita su diagnóstico. Además, este desplazamiento produce disincronía de contracción en las cavidades derechas, ya que la contracción de la pared lateral del ventrículo derecho es tardía en relación al tabique. Es a través de este mecanismo que se produce bajo gasto cardiaco derecho. La detección del desplazamiento septal sistólico a las cavidades izquierdas causa interdependencia ventricular; es un hallazgo ecocardiográfico en pacientes con SIRA que hace el diagnóstico de falla ventricular derecha aguda ${ }^{66}$.

\section{Gasto cardiaco derecho en pacientes con SIRA y falla cardiaca derecha aguda}

El volumen anterógrado a través de la válvula pulmonar tiene una relación estricta en relación al tiempo del ciclo de ventilación mecánico en que se encuentra. El volumen anterógrado que nos ayuda a calcular el gasto cardiaco derecho será menor durante la fase inspiratoria en la ventilación mecánica positiva, y será mayor durante el ciclo espiratorio, por lo que el cálculo del gasto cardiaco derecho determinado por el índice tiempo/velocidad en el tracto de salida del ventrículo derecho debe ser medido de preferencia en la fase espiratoria para no realizar cálculos erróneos ${ }^{53}$.

\section{Conclusiones}

Durante las crisis emergentes se puede trabajar sin la estadística mientras esta se va construyendo, pero nunca sin fisiología y unidad (protocolos). La explicación fisiológica aquí vertida es lo más exacta posible.
El protocolo es una inducción derivada de dicha fisiología en espera de tener pronto una estadística que nos diga si lo que hacemos es de utilidad o no. Seguir protocolos bien estructurados puede ayudar a que rápidamente nos demos cuenta de en qué estamos equivocados, así como de la dirección y magnitud de nuestro error. En conclusión, lo que el lector tiene en sus manos son conjeturas en búsqueda de refutaciones. Al momento de escribir este artículo, la única respuesta correcta es «aún no lo sabemos».

\section{Agradecimientos}

A la Academia Mexicana de Cirugía, al Colegio Mexicano de Medicina Crítica y al Colegio Mexicano de Anestesiología, por su apoyo y participación en este trabajo de revisión.

Al Dr. Paolo Pelosi por sus enseñanzas en ventilación mecánica, que forman parte de este texto. ¡Fuerza, Italia!

A todo el gremio médico que en esta pandemia de COVID-19 permanece con gran resiliencia a la adversidad y busca lo mejor para el cuidado de los pacientes.

\section{Conflicto de intereses}

Los autores declaran no tener conflicto de intereses para este texto.

\section{Financiamiento}

No se recibió financiamiento para este trabajo.

\section{Responsabilidades éticas}

Protección de personas y animales. Los autores declaran que para esta investigación no se han realizado experimentos en seres humanos ni en animales.

Confidencialidad de los datos. Los autores declaran que han seguido los protocolos de su centro de trabajo sobre la publicación de datos de pacientes.

Derecho a la privacidad y consentimiento informado. Los autores declaran que en este artículo no aparecen datos de pacientes.

\section{Bibliografía}

1. Zhang $\mathrm{H}$, Penninger JM, Li Y, Zhong N, Slutsky AS. Angiotensin-converting enzyme 2 (ACE2) as a SARS-CoV-2 receptor: molecular mechanis$\mathrm{ms}$ and potential therapeutic target. Intensive Care Med. 2020;46:586-90. 
2. Ruan Q, Yang K, Wang W, Jiang L, Song J. Clinical predictors of mortality due to COVID-19 based on an analysis of data of 150 patients from Wuhan, China. Intensive Care Med. 2020;46:1294-7.

3. Zhou G, Zhao Q. Perspectives on therapeutic neutralizing antibodies against. The novel coronavirus SARS-CoV-2. Int J Biol Sci. 2020;16:1718-23.

4. Frat JP, Thille A, Mercat A, Girault C, Ragot S, Perbet S, et al High-flow oxygen through nasal cannula in acute hypoxemic respiratory failure. $\mathrm{N}$ Engl J Med. 2015;372:2185-96.

5. The ARDS Definition Task Force. Acute respiratory distress syndrome. The Berlin Definition. JAMA. 2012;307:2526-33.

6. Gattinoni L, Chiumello D, Caironi P, Busana M, Romitti F, Brazzi L, et al. COVID-19 pneumonia: different respiratory treatment for different phenotypes? Intensive Care Med. 2020;46:1099-102.

7. Zhu N, Zhang D, Wang W, Li X, Yang B, Song J, et al.; China Novel Coronavirus Investigating and Research Team. A novel coronavirus from patients with pneumonia in China, 2019. N Engl J Med. 2020;382:727-33.

8. Li Q, Guan X, Wu P, Wang X, Zhou L, Tong Y, et al. Early transmission dynamics in Wuhan, China, of novel coronavirus infected pneumonia. N Engl J Med. 2020;382:1199-207.

9. Chan JF, Yuan S, Kok KH, To KK, Chu H, Yang J, et al. A familial cluster of pneumonia associated with the 2019 novel coronavirus indicating person-to-person transmission: a study of a family cluster. Lancet. 2020;395:514-23.

10. Wang D, Hu B, Hu C, Zhu F, Liu X, Zhang J, et al. Clinical characteristics of 138 hospitalized patients with 2019 novel coronavirusロinfected pneumonia in Wuhan, China. JAMA. 2020;323:1061-9.

11. Wu Z, McGoogan JM. Characteristics of and important lessons from the coronavirus disease 2019 (COVID19) outbreak in China: summary of a report of 72314 cases from the Chinese center for disease control and prevention. JAMA. 2020 Feb 24. doi: 10.1001/jama.2020.2648. Online ahead of print.

12. Tanigawa Y, Rivas M. Initial review and analysis of COVID-19 host genetics and associated phenotypes. Preprints 2020. doi:10.20944/preprints202003.0356.v1

13. Qin C, Zhou L, Hu Z, Zhang S, Yang S, Tao Y, et al. Dysregulation of immune response in patients with COVID-19, Wuhan, China. Clin Infect Dis. 2020 Mar 12:ciaa248. doi: 10.1093/cid/ciaa248. Online ahead of print.

14. Zhao J, Wu Y, Feng Z, Li P, Yu Q. Relationship between the ABO blood group and the COVID-19 susceptibility. medRxiv 2020. doi: https://doi.or g/10.1101/2020.03.11.20031096.

15. Mehta P, McAuley DF, Brown M, Sanchez E, Tattersall RS, Manson JJ HLH Across Speciality Collaboration, UK. COVID-19: consider cytokine storm syndromes and immunosuppression. Lancet. 2020:395:1033-4.

16. Novel Coronavirus Pneumonia Emergency Response Epidemiology Team. The epidemiological characteristics of an outbreak of 2019 nove coronavirus disease (COVID-19) - China, 2020. China CDC Weekly. (Consultado el 16 de marzo de 2020.) Disponible en: http://weekly.chinacdc.cn/en/article/id/e53946e2-c6c4-41e9-9a9b-fea8db1a8f51.

17. Wan Y, Shang J, Graham R, Baric RS, Li F. Receptor recognition by novel coronavirus from Wuhan: an analysis based on decade-long structural studies of SARS. J Virol. 2020;94:e00127-20.

18. Wang M, Cao R, Zhang L, Yang X, Liu J, Xu M, et al. Remdesivir and chloroquine effectively inhibit the recently emerged novel coronavirus (2019-nCoV) in vitro. Cell Res. 2020;30:269-71.

19. Siddiqi HK, Mehra MR. COVID-19 illness in native and immunosuppressed states: a clinical-therapeutic staging proposal. J Heart Lung Transplant. 2020;39:405-7.

20. Rodríguez AJ, Cardona O, Gutiérrez O, Villamizar R, Holguin Y, Escalera JP, et al. Latin American Network of Coronavirus Disease 2019-COVID-19 Research (LANCOVID-19). Clinical, laboratory and imaging features of COVID-19: a systematic review and meta-analysis. Travel Med Infect Dis. 2020;34:101623.

21. Marik P. MATH protocol covid-19 management protocol EVMS. Consultado: marzo 2020. Disponible en: https://www.evms.edu/media/evms_public/departments/internal_medicine/EVMS_Critical_Care_COVID-19_ Protocol.pdf

22. Ospina-Tascón GA, Bautista DF, Madriñán HJ, Valencia JD, Bermúdez WF, Quiñones E, et al. Microcirculatory dysfunction and dead-space ventilation in early ARDS: a hypothesis-generating observational study. Ann Intensive Care. 2020;24;10.

23. The National Heart, Lung, and Blood Institute ARDS Clinical Trials Network Higher versus lower positive end-expiratory pressures in patients with the acute respiratory distress syndrome. N Engl J Med. 2004;351:327-36.

24. Papazian L, Aubron C, Brochard L, Chiche JL, Combes A, Didier Dreyfuss $D$, et al. Formal guidelines: management of acute respiratory distress syndrome. Ann Intensive Care. 2019;9:69.

25. Scaramuzzo G, Ball L, Pino F, Ricci L, Larsson A, Guérin C, et al. Influence of positive end-expiratory pressure titration on the effects of pronation in acute respiratory distress syndrome: a comprehensive experimental study. Front Physiol. 2020;11:179.

26. Alveolar Recruitment for Acute Respiratory Distress Syndrome Tria (ART) Investigators. Effect of lung recruitment and titrated positive end-expiratory pressure (PEEP) vs low peep on mortality in patients with acute respiratory distress syndrome. A randomized clinical trial. JAMA. 2017;318:1335-45

27. Mekontso Dessap A, Boissier F, Charron C, Bégot E, Repessé X, Legras A, et al. Acute cor pulmonale during protective ventilation for acute respiratory distress syndrome: prevalence, predictors, and clinical impact. Intensive Care Med. 2016;42:862-70.

28. Bertoni M, Spadaro S, Goligher. Monitoring patient respiratory effort during mechanical ventilation: lung and diaphragm-protective ventilation. Crit Care. 2020;24:106.

29. Gage A, Higgins A, Ran Lee, Siyab P. Reacquainting cardiology with mechanical ventilation in response to the COVID-19 pandemic. JACC Case Rep. 2020 Mar 27. doi: 10.1016/j.jaccas.2020.03.007. Online ahead of print

30. Munshi L, Walkey A, Goligher E, Pham T, Uleryk EM, Fan E. Venovenous extracorporeal membrane oxygenation for acute respiratory distress syndrome: a systematic review and meta-analysis. Lancet Respir Med. 2019;7:163-72.

31. Rochwerg B, Brochard L, Elliott MW, Hess D, Hill NS, Nava S, et al. Official ERS/ATS clinical practice guidelines: noninvasive ventilation for acute respiratory failure. Eur Respir J. 2017;50:1602426.

32. Wu C, Chen X, Cai Y, Xia J, Zhou X, Xu S, et al. Risk factors associated with acute respiratory distress syndrome and death in patients with coronavirus disease 2019 pneumonia in Wuhan, China. JAMA Intern Med. 2020;180:1-11.

33. Branson RD, Rubinson L. One ventilator, multiple patients: what the data really supports. Resuscitation. 2008:79:17102; author reply $172 \square 3$

34. Joint Statement on Multiple Patients Per Ventilator SCCM, AARC, ASA, APSF, AACN, and CHEST Share Unified Message. Disponible en: https://www.aarc.org/wp- content/uploads/2020/03/032620-COVID-19press-release.pdf.

35. Gatinoni L, Busana M, Giosa L, Macrì MM, Quintel M. Prone positioning in acute respiratory distress syndrome. Semin Respir Crit Care Med. 2019;40:94-100.

36. Pan C, Chen L, Lu C, Zhang W, Xia JA, Sklar MC, et al. Lung recruitability in SARS-CoV-2 associated acute respiratory distress syndrome: a single-center, observational study. Am J Respir Crit Care Med. 2020;201:1294-7.

37. Sun, Qiu H, Huang M, Yang Y. Lower mortality of COVID-19 by early recognition and intervention: experience from Jiangsu Province. Ann Intensive Care. 2020;10:33.

38. Scaravilli V, Scaravilli V, Grasselli G, Castagna L, Zanella A, Isgrò S, Lucchini $A$, et al. Prone positioning improves oxygenation in spontaneously breathing nonintubated patients with hypoxemic acute respiratory failure: a retrospective study. J Crit Care. 2015;30:1390-4.

39. Kumar TC, Shivakumar NS, Deepak TS, Krishnappa R, Goutam MS, Ganigar V. H1N1-infected Patients in ICU and Their Clinical Outcome. N Am J Med Sci. 2012;4(9):394-8.

40. Gattinoni L, Chiumello D, Caironi P, Busana M, Romitti F, Brazzi L, et al. COVID-19 pneumonia: different respiratory treatment for different phenotypes? Intensive Care Med. 2020:46:1099-102.

41. Claure-Del Granado R, Mehta RL. Fluid overload in the ICU: evaluation and management. BMC Nephrol. 2016;17:109.

42. Mehta P, McAuley DF, Brown M, Sanchez E, Tattersall RS, Manson JJ. COVID-19: consider cytokine storm syndromes and immunosuppression. Lancet. 2020;395:1033-4.

43. Zeng J, Huang J, Pan L. How to balance acute myocardial infarction and COVID-19: the protocols from Sichuan Provincial People's Hospital. Intensive Care Med. 2020;46:1111-3.

44. Shi S, Qin M, Shen B, Cai Y, Liu T, Yang F, et al. Association of cardiac injury with mortality in hospitalized patients with COVID-19 in Wuhan, China. JAMA Cardiol. 2020 Mar 25:e200950. doi: 10.1001/jamacardio.2020.0950. Online ahead of print.

45. Schunkert $H$, Erbel $R$. [Evidence-based primary prevention: where do we stand in 2020?] Herz. 2020;45:1-2.

46. Sondergaard S. Pavane for a pulse pressure variation defunction. Crit Care. 2013:17:327.

47. Vieillard-Baron A, Chergui K, Augarde R, Prin S, Page B, Beauchet A, et al. Cyclic changes in arterial pulse during respiratory support revisited by Doppler echocardiography. Am J Respir Crit Care Med. 2003;168:671-6.

48. Grissom CK, Hirshberg EL, Dickerson JB, Brown SM, Lanspa MJ, Liu KD, et al. Fluid management with a simplified conservative protocol for the acute respiratory distress syndrome. Crit Care Med. 2015;43:288-95.

49. Wilson JG, Calfee CS. ARDS subphenotypes: understanding a heterogeneous syndrome. Crit Care. 2020;24):102

50. Famous KR, Delucchi K, Ware LB, Kangelaris KN, Liu KD, Thompson T, et al. Acute respiratory distress syndrome subphenotypes respond differently to randomized fluid management strategy. Am J Respir Crit Care Med. 2017; 195:331-8

51. Tourneux P, Rakza T, Bouissou A, Krim G, Storme L. Pulmonary circulatory effects of norepinephrine in newborn infants with persistent pulmonary hypertension. J Pediatr. 2008;153:345-9.

52. Mizota T, Fujiwara K, Hamada M, Matsukawa S, Segawa H. Effect of arginine vasopressin on systemic and pulmonary arterial pressure in a 
patient with pulmonary hypertension secondary to pulmonary emphysema: a case report. JA Clin Rep. 2017;3:1.

53. Repessé X, Vieillard-Baron A. Right heart function during acute respiratory distress syndrome. Ann Transl Med. 2017;5:295.

54. Ferraris A, Bouisse C, Mottard N, Thiollière F, Anselin S, Piriou V, et al Mottling score and skin temperature in septic shock: relation and impact on prognosis in ICU. PLoS One. 2018;13:e0202329.

55. Pickard A, Karlen W, Ansermino JM. Capillary refill time: is it still a useful clinical sign? Anesth Analg. 2011;113:120-3.

56. Berridge JC. Influence of cardiac output on the correlation between mixed venous and central venous oxygen saturation. $\mathrm{Br} \mathrm{J}$ Anaesth. 1992:69:409-10.

57. Mallat J, Lemyze M, Tronchon L, Vallet B, Thevenin D. Use of venous-to-arterial carbon dioxide tension difference to guide resuscitation therapy in septic shock. World J Crit Care Med. 2016;5:47-56.

58. Tang KQ, Yang SL, Zhang B, Liu HX, Ye DY, Zhang HZ, et al. Ultrasonic monitoring in the assessment of pulmonary recruitment and the best positive end-expiratory pressure. Medicine (Balt.). 2017;96:e8168.

59. Bouhemad B, Liu ZH, Arbelot C, Zhang M, Ferarri F, Le-Guen M, et al Ultrasound assessment of antibiotic-induced pulmonary reaeration in ventilator-associated pneumonia. Crit Care Med. 2010;38:84-92.

60. Bouferrache K, Vieillard-Baron A. Acute respiratory distress syndrome, mechanical ventilation, and right ventricular function. Curr Opin Crit Care.
2011;17:30-5.

61. Brower RG, Matthay MA, Morris A, Schoenfeld D, Thompson BT, et al. Acute respiratory distress syndrome network ventilation with lower tidal volumes as compared with traditional tidal volumes for acute lung injury and the acute lung injury and the acute respiratory distress syndrome. N Engl J Med. 2000;342:1301-8.

62. Jardin F, Vieillard-Baron A. Is there a safe plateau pressure in ARDS? The right heart only knows. Intensive Care Med. 2007:33:444-7.

63. Bello G, Blanco P. Lung ultrasonography for assessing lung aeration in acute respiratory distress syndrome: a narrative review. J Ultrasound Med. 2019;38:27-37

64. Lang RM, Badano LP, Mor-Avi V, Afilalo J, Armstrong A, Ernande L, et al. Recommendations for cardiac chamber quantification by echocardiography in adults: an update from the American Society of Echocardiography and the European Association of Cardiovascular Imaging. $J$ Am Soc Echocardiogr. 2015;28:1-39.e14.

65. Biswas A. Right heart failure in acute respiratory distress syndrome: an unappreciated albeit a potential target for intervention in the management of the disease. Indian J Crit Care Med. 2015;19:606-9.

66. Mizota T, Fujiwara K, Hamada M, Matsukawa S, Segawa H. Effect of arginine vasopressin on systemic and pulmonary arterial pressure in a patient with pulmonary hypertension secondary to pulmonary emphysema: a case report. JA Clin Rep. 2017;3:1. 